\title{
Mejora del proceso de aprendizaje de la escritura mediante las rutinas del pensamiento
}

Improvement of the learning process of writing through the routines of thought

\section{Raúl Gutiérrez-Fresneda \\ Universidad de Alicante \\ España}

\section{Raquel Gilar Corbí \\ Universidad de Alicante \\ España}

ONOMÁZEIN 53 (septiembre de 2021): 192-209

DOI: 10.7764/onomazein.53.10

ISSN: 0718-5758

\section{(c) $(1) \Theta$}

Raúl Gutiérrez-Fresneda: Departamento de Psicología Evolutiva y Didáctica, Facultad de Educación, Universidad de Alicante, España. | E-mail: raul.gutierrez@ua.es

Raquel Gilar Corbí: Departamento de Psicología Evolutiva y Didáctica, Facultad de Educación, Universidad de Alicante, España. | E-mail: raquel.gilar@ua.es 


\section{Resumen}

La adquisición de la escritura requiere de la intervención de procesos cognitivos de gran complejidad, lo que origina dificultades en su aprendizaje desde las primeras edades. La puesta en común de los conocimientos, ideas e hipótesis personales que va construyendo cada estudiante a través de propuestas que potencien la reflexión cognitiva sobre el proceso de construcción de la escritura puede ser una estrategia de gran utilidad para el dominio de este aprendizaje. El objetivo de este trabajo fue analizar el efecto que la intervención de un programa centrado en el desarrollo de las habilidades que favorecen el acceso al sistema de la escritura mediante dinámicas que potencien la capacidad de pensar a través de las rutinas de pensamiento presenta en el proceso de adquisición de la escritura. Se empleó un diseño cuasi-experimental de comparación entre grupos con medidas pretest y postest. En el estudio participaron 356 estudiantes con edades entre los 5 y 6 años. Los resultados ponderan el valor potencial del programa y apoyan el desarrollo de modelos de enseñanza que integren las habilidades del pensamiento en la práctica del aula para la mejora del sistema de la escritura en los primeros niveles escolares.

Palabras clave: escritura; competencias de escritura; desarrollo cognitivo; rutinas del pensamiento; enseñanza de lenguas.

\section{Abstract}

The learning of writing requires the intervention of cognitive processes of great complexity, which causes difficulties in their acquisition from early ages. The sharing of knowledge, ideas, and personal hypotheses that each student is building through proposals that enhance cognitive reflection on the process of construction of writing can be a very useful strategy for mastering this learning. The objective of this work was to analyze the effect that the intervention of a program focused on the development of skills that favor access to the writing system through dynamics that enhance the ability to think and reason in a shared way through the routines of thought presents in the process of acquisition of writing. A quasi-experimental design of comparison between groups with pretest and posttest measurements was used. The study involved 356 students aged between 5 and 6 years. The results weigh the potential value of the program and support the development of teaching models that integrate thinking skills into classroom practice for the improvement of the writing system in the first school levels.

Keywords: writing skills; cognitive development; routines of thought; language teaching. 


\section{Introducción}

El aprendizaje de la escritura es uno de los retos principales al que se tienen que enfrentar los niños durante los primeros niveles de escolaridad. Una gran parte logra su dominio sin demasiadas dificultades, pero existe un porcentaje elevado de estudiantes que presentan serias dificultades en su aprendizaje. Uno de los aportes fundamentales de los últimos años respecto al proceso de aprendizaje de la escritura ha sido conocer las relaciones existentes entre el lenguaje oral y el aprendizaje de la lengua escrita (Bravo, 2016; Defior y Serrano, 2011; Dickinson, 2011; Díez, Güemes y Molina, 2018; Gutiérrez, 2018; Llamazares, Alonso-Cortés y Sánchez, 2015). Para adquirir el lenguaje escrito en un sistema alfabético como es el castellano se precisa acceder al conocimiento metalingüístico, por lo que se ha de saber que nuestro sistema de representación de la lengua se basa en la división de las palabras en sus segmentos fonológicos, así como tomar conciencia de la estructura fonológica del lenguaje (González, López, Vilar y Rodríguez, 2013; González, Cuetos, Vilar y Uceira, 2015; Gutiérrez, 2017). Comprender que los símbolos escritos representan a los fonemas del lenguaje hablado es una tarea que requiere de un proceso de reflexión y análisis cognitivo de gran complejidad por su elevado carácter abstracto. Ahora bien, la función real de la escritura como actividad es favorecer la comprensión, comunicación y recreación del mundo. En otras palabras, escribir no es una actividad mecánica, sino una práctica sociocultural con su propia estructura, sus propias transformaciones internas y su propio desarrollo.

Cuando los escolares acceden al aprendizaje de la lengua escrita han de saber que nuestro sistema de representación se basa en la segmentación de la cadena hablada y que las letras son signos gráficos que se corresponden con los elementos sonoros, ya que cada letra se asocia con una unidad de sonido (Defior y Serrano, 2011; Gutiérrez, 2017). La capacidad para analizar los elementos sonoros de las palabras se conoce como conciencia fonológica y para su logro se requiere del desarrollo de las habilidades fonológicas ya que mediante su desarrollo se facilita la relación entre ambos lenguajes (oral y escrito). La conciencia fonológica no constituye un fenómeno unitario, sino que se pueden distinguir varios niveles dependiendo de las unidades de segmentación: conciencia léxica, silábica, intrasilábica y fonémica. De todos ellos, existe acuerdo en que el manejo de las unidades mínimas de las palabras y la capacidad de descubrir la secuencia de los fonemas que las componen son los aspectos que más relación presentan con el aprendizaje de la lengua escrita (Gutiérrez y Díez, 2018).

El conocimiento alfabético es otro componente importante del aprendizaje del código escrito, lo cual es lógico ya que en un sistema de representación como el español la relación entre los nombres de las letras y sus sonidos facilita la adquisición de los procesos de correspondencia fonema-grafema (Schatsneider, Fletcher, Francis, Carlson y Foorman, 2004; Gutiérrez y Díez, 2018). Estudios correlacionales han demostrado la fuerte relación existente entre el nombre y/o el sonido de las letras y el dominio del código escrito (Bravo, Villalón y Orellana, 2006). Del mismo modo se ha constatado que el conocimiento alfabético favorece el desarro- 
Ilo de habilidades fonológicas, al establecerse una relación causal entre el conocimiento del nombre de las letras y el aprendizaje de los sonidos de estas (Share, 2004).

Igualmente existe consenso en la literatura respecto a la necesidad de promover el desarrollo del pensamiento crítico en el ámbito escolar (Boodt, 1984; Norris, 1985; Rapp, 1988) ya que los cambios sociales exigen individuos competentes que sean creativos, críticos, autónomos, con iniciativa personal, capaces de tomar decisiones, por lo que es necesario que desde el ámbito escolar se prepare a los estudiantes a dar respuesta de manera eficaz a los problemas del entorno cotidiano. Para contribuir a ello, es preciso enseñar a pensar de manera crítica en el aula con la finalidad de ayudar a los estudiantes a ofrecer respuestas creativas y eficaces adecuadas a las demandas escolares y sociales (Gallardo y Novillo, 2017; Gutiérrez-Fresneda, 2018; Swartz y Perkins, 1989). Sin embargo, la realidad es que actualmente son escasos los trabajos efectuados con la finalidad de analizar las aportaciones que las habilidades del pensamiento pueden ofrecer a los contenidos de las asignaturas del currículum. Aunque, afortunadamente, en los últimos años han comenzado a realizarse algunas investigaciones con estudiantes de cursos superiores en las que se han evidenciado los beneficios que las habilidades del pensamiento presentan en los procesos de aprendizaje de diferentes materias escolares (Araya, 2014; Aznar y Laiton, 2017; Sáiz y Fernández, 2012). Respecto al lenguaje escrito también se han efectuado recientemente algunos trabajos (Gutiérrez-Fresneda y Díez-Mediavilla, 2017; Gutiérrez-Fresneda, 2018) en los que se han puesto de manifiesto los beneficios que las habilidades del pensamiento presentan en la mejora de la composición escrita de los estudiantes que han adquirido los procesos cognitivos inferiores del sistema de la escritura.

El auge por el desarrollo de una cultura del pensamiento en el aula se ha concretado a través del diseño de una serie de herramientas cognitivas como es el caso de las rutinas del pensamiento, las cuales han contribuido a facilitar la adquisición de diversos aprendizajes (Báez y Onrubia, 2016; Beas, Santa Cruz, Thomsen y Utreras, 2001; Gallardo y Novillo, 2017; Toro y Arguis, 2015), siendo en la actualidad un recurso pedagógico de gran utilidad para generar ideas, potenciar la capacidad de razonar, fomentar la reflexión y comprender con mayor eficacia los contenidos escolares (Gallardo y Novillo, 2017; Swartz y Perkins, 1989; Valenzuela, 2007). Las rutinas del pensamiento permiten hacer visible los conocimientos, ideas, reflexiones e hipótesis que los estudiantes realizan, por lo que pueden constituir un instrumento eficaz para el acceso al sistema de la escritura en las primeras edades, en cuanto que favorecen la creación de hábitos mentales reflexivos, la generación de ideas, la organización de acciones mentales, el establecimiento de relaciones, así como la estructuración coherente de los contenidos aprendidos, todo lo cual está en relación directa con la teoría psicogenética de la escritura, que considera que este aprendizaje es el resultado de un proceso de reconstrucción cognitiva que permite al estudiante descubrir las características del sistema de representación del código escrito (Ferreiro, 1979; Ferreiro y Teberosky, 1988; Nemirovsky, 1999; Teberosky y Portilla, 2011). 
Desde el enfoque funcionalista, el acto escritor no es una actividad centrada exclusivamente en la simple construcción y representación de grafías y palabras, sino un medio de construcción cultural que tiene lugar en situaciones comunicativas significativas a través de la interacción con los otros (Graham, 2006; Gutiérrez, 2017; Montesdeoca, Gómez-Parra, y Espejo, 2020). Diferentes trabajos llevados a cabo en centros educativos señalan que una gran parte de las acciones pedagógicas relacionadas con la escritura se caracterizan por ser prácticas de carácter repetitivo y mecánico que reflejan la ausencia de actividades de tipo cognitivo y metacognitivo (Peña y Quintero, 2016), de aquí la importancia de la puesta en práctica de dinámicas que fomenten la interacción conjunta y que a su vez contribuyan al desarrollo del pensamiento crítico.

La relevancia de la adquisición de un buen aprendizaje de la escritura en las primeras edades es evidente; sin embargo, a pesar de las aportaciones de los diferentes trabajos realizados hasta la fecha, se sigue encontrando un gran número de estudiantes que presentan problemas en el dominio de esta habilidad lingüística desde los inicios de su aprendizaje, lo que sugiere la necesidad de seguir profundizando en metodologías innovadoras que contribuyan a la mejora de los procesos de aprendizaje de la escritura con la finalidad de favorecer su puesta en práctica en la dinámica del aula. Son varias las experiencias en las que se han puesto de manifiesto los aportes que las habilidades de pensamiento ofrecen al aprendizaje de los estudiantes, especialmente de cursos superiores (Gallardo y Novillo, 2017; Gutiérrez-Fresneda y Díez, 2018). Sin embargo, no se encuentran trabajos en los que se constaten los beneficios que el desarrollo de las rutinas del pensamiento puede presentar en el acceso al aprendizaje del lenguaje escrito en las primeras edades.

El objetivo de este estudio fue analizar el efecto que la intervención de un programa centrado en el desarrollo de las habilidades que favorecen el aprendizaje de la escritura mediante dinámicas que potencien la capacidad de pensar y razonar de manera compartida a través de las rutinas de pensamiento presenta en el proceso de adquisición de la escritura. Para ello, se compara el grado de adquisición de la escritura en dos muestras de alumnos de entre 5 y 6 años, uno que recibe intervención en este proceso de aprendizaje mediante la utilización de las rutinas de pensamiento y otro que sigue el mismo programa de enseñanza de la escritura a través de la clase magistral. La hipótesis que se plantea es que los estudiantes que participan en el programa de aprendizaje de la escritura mediante el apoyo de las rutinas de pensamiento obtendrán un mejor dominio del código escrito.

\section{Método}

\subsection{Participantes}

En el estudio participaron 356 alumnos con edades comprendidas entre los 5 y 6 años que cursaban el tercer curso de Educación Infantil $(M=5.37$; DT = 0.42), de los cuales el 49.3\% eran 
niños y el 50.7\% niñas. Para la elección de la muestra se eligieron al azar cuatro colegios públicos y concertados ubicados en zonas de nivel sociocultural medio de la provincia de Alicante (España), formándose dos grupos: Ios que aplicarían voluntariamente el programa de intervención (grupo experimental) y los que no aplicarían el programa (grupo control). La asignación de los centros a los distintos niveles de tratamiento se realizó aleatoriamente antes de evaluar al alumnado, quedando un colegio público y otro concertado en el grupo experimental (180 estudiantes), lo mismo que en el grupo control (176 estudiantes). De los 180 participantes experimentales, el $48.6 \%$ son varones y el $51.4 \%$ mujeres, mientras que de los 176 participantes del grupo control, el $49.6 \%$ son varones y el $50.4 \%$ son mujeres. En cada grupo de estudio se eligieron a aquellos sujetos de habla castellana que no presentaban alteraciones físicas, psíquicas o sensoriales y que tenían un nivel intelectual normal. El análisis de contingencia (chi cuadrado de Pearson) entre condición y sexo no evidenció diferencias estadísticamente significativas $\left(X^{2}=0.52, p>05\right)$.

\subsection{Instrumentos de evaluación}

Con la finalidad de evaluar las variables dependientes objeto de estudio se utilizaron tres instrumentos de evaluación con garantías psicométricas de fiabilidad y validez.

- Prueba de Lenguaje Oral Navarra Revisada (PLON-R). Para la evaluación del lenguaje oral se empleó la prueba PLON-R (Aguinaga, Armentia, Fraile, Olangua y Útiz, 2004). Se trata de una prueba estandarizada destinada a niños de entre tres y seis años que tiene por finalidad analizar los distintos componentes del lenguaje: forma (fonología, morfología y sintaxis), contenido (semántica) y uso (pragmática). Las puntuaciones directas de cada una de las dimensiones se transforman en puntuaciones típicas organizadas en tres categorías: "retraso", "necesita mejorar" y "normal”. Dicha prueba según los datos obtenidos por los autores presenta un coeficiente de fiabilidad de Cronbach de 87 .

- Prueba para la Evaluación del Conocimiento Fonológico (PECO) (Ramos y Cuadrado, 2006). Este test evalúa dos niveles de conocimiento fonológico (silábico y fonémico), cada uno de los cuales se compone de tres tareas distintas: identificación, adición y omisión. Además, tiene en cuenta la posición que ocupa la sílaba o el fonema con el que se trabaja: al inicio, en medio o al final de la palabra. Esta prueba incluye tres subtest con sílabas y fonemas (tareas de identificación, adición y omisión), con un total de 30 ítems (15 de sílabas y 15 de fonemas). La confiabilidad, estimada por los autores a través del coeficiente alfa de Cronbach, es de .80.

- Proescri-Primaria (Prueba de Evaluación de los Procesos Cognitivos en la Escritura) (Artiles y Jiménez, 2007). Para la valoración del grado de adquisición del aprendizaje de la escritura se utilizaron las pruebas relativas al dominio de los procesos léxicos, los cuales incluyen las tareas de dictado de letras, generar palabras, dictado de palabras, 
dictado de palabras sujetas a reglas ortográficas y dictado de pseudopalabras. En cada una de las pruebas se concede un punto por cada respuesta correcta. De acuerdo con el procedimiento de Cronbach realizado por los autores de la prueba, el coeficiente de fiabilidad es de 85 .

\subsection{Diseño y procedimiento}

El estudio utilizó un diseño cuasi-experimental de medidas repetidas pretest-postest con grupo control. Antes de iniciarse el programa de trabajo y a su finalización, se aplicaron tres instrumentos de evaluación a los alumnos experimentales y control mediante las pruebas PLON-R, PECO y Proescri, con el propósito de medir las variables dependientes sobre las que se hipotetizó que el programa iba a tener efecto: lenguaje oral, conciencia fonológica y conocimiento alfabético, ya que se ha demostrado que estas habilidades influyen positivamente en el proceso de aprendizaje de la escritura. La aplicación de las pruebas tanto antes como después de implementar el programa fue llevada a cabo por profesionales de la educación previamente entrenados, lo que facilitó la homogeneidad en la recogida de los datos.

La valoración inicial de los alumnos se realizó de forma individual en espacios próximos al aula ordinaria durante el mes de enero dentro del horario escolar. Con posterioridad se puso en práctica el programa de intervención en el grupo experimental, el cual tuvo una duración de 20 sesiones (4 sesiones de 50 minutos durante 5 semanas), el mismo tiempo que el grupo control. Al finalizar el segundo trimestre del curso, momento en el que ya se había aplicado por completo el programa, se volvió a realizar la evaluación a todos los participantes con los mismos instrumentos. El estudio respetó los valores éticos requeridos en la investigación con personas (consentimiento informado, derecho a la información, protección de datos personales, garantías de confidencialidad, no discriminación, gratuidad y tener la posibilidad de abandonar el programa en cualquiera de sus fases).

\subsection{Programa de intervención}

El programa de intervención tenía como objetivo desarrollar de manera explícita el lenguaje oral, la conciencia fonológica y el conocimiento alfabético mediante el apoyo de diferentes rutinas del pensamiento. Durante el periodo de intervención el alumnado del grupo control siguió el mismo programa de trabajo que los estudiantes del grupo experimental ejercitando de manera explícita el lenguaje oral, la conciencia fonológica y el conocimiento alfabético, con la diferencia únicamente entre ambos grupos en el modo de implementar el programa de trabajo.

En el grupo control el profesor inicialmente explicaba los aprendizajes a la clase, se seguía con la realización de una serie de actividades en equipos de trabajo relacionadas con el contenido correspondiente finalizándose con la corrección del trabajo individualmente por parte del profesor tutor. Por otro lado, a los estudiantes pertenecientes al grupo experimental se les explicaba el contenido de manera colectiva mediante una rutina del pensamiento; a con- 
tinuación, el alumnado realizaba las actividades propuestas en pequeños grupos moderados por un compañero coordinador (capitán), quien destacaba por sus habilidades sociales y comunicativas. Su labor se centraba en dinamizar el proceso de trabajo mediante el empleo de un organizador visual y la formulación de una serie de cuestiones guía, las cuales estaban relacionadas con la rutina de pensamiento presentada.

Para garantizar su adecuado funcionamiento, el docente previamente había explicado y puesto en práctica con los responsables de cada grupo la dinámica a realizar, y durante su puesta en práctica supervisaba su correcto desarrollo. Las rutinas de trabajo empleadas fueron: veo pienso - me pregunto, palabra-frase-idea, titular, color-símbolo-imagen, antes pensaba - ahora pienso y problema-solución, a partir de las cuales se planteaban distintas cuestiones con la finalidad de potenciar la capacidad de pensar tanto de manera individual como colectiva con los compañeros del grupo. Esto permitía la generación de ideas, la verbalización conjunta y la reflexión compartida sobre las habilidades preescritoras que conformaban el programa de intervención, lo que contribuía a poner en común las aportaciones personales y enriquecer las hipótesis y aprendizajes que los compañeros iban realizando sobre el proceso de construcción del sistema de la escritura.

Para el desarrollo del lenguaje oral a partir de los organizadores visuales y la formulación de preguntas se ejercitaron los componentes lingüísticos relativos a la forma, contenido y uso. Los componentes fonológico, morfológico y sintáctico (forma) se trabajaron mediante onomatopeyas, denominación de ilustraciones, actividades de completar frases a partir de una serie de palabras, construir oraciones mediante una serie de imágenes dadas, invención de títulos de cuentos y creación conjunta de pequeños textos narrativos. Por ejemplo, mediante la rutina de pensamiento "titular" se solicitaba a los estudiantes que a partir de una ilustración pensaran el título de un cuento. El componente semántico (contenido) se ejercitó con tareas de encadenamientos mediante la utilización de imágenes del entorno próximo y del contenido de los textos trabajados a través de juegos de búsqueda de palabras del contexto presente y ausente, clasificación de objetos cotidianos en campos semánticos, identificación de palabras intrusas en oraciones y búsquedas de sinónimos y antónimos. Se utilizó entre otras la rutina de pensamiento "palabra-frase-idea”, en la que a partir de una palabra trabajada se solicitaba la elaboración de una frase y la creación de una idea (ej.: palabra zanahoria, frase En la frutería venden zanahorias; para la generación de la idea se indicaba una finalidad, "para qué sirven": Las zanahorias son buenas para la vista). Mientras que la capacidad comunicativa (uso) que tenía como finalidad favorecer la utilización del lenguaje funcional se desarrolló mediante dinámicas orientadas a la expresión de situaciones comunicativas a partir de la temática de los textos narrativos trabajados, así como mediante diferentes situaciones comunicativas vinculadas con experiencias y vivencias personales.

La conciencia fonológica se ejercitó con la ayuda de las rutinas del pensamiento mediante actividades orientadas al desarrollo de la conciencia silábica y fonémica tomadas de los 
materiales Avanza (Espejo, Gutiérrez, Llambés y Vallejo, 2008) y Avanzados (Espejo, Gutiérrez, Llambés y Vallejo, 2015), tanto por su carácter lúdico y motivador como por haber sido referentes de investigaciones precedentes (Gutiérrez-Fresneda, Díez y Jiménez-Pérez, 2017). Las diferentes propuestas se centraron en favorecer la identificación del número de sílabas en palabras de diferente longitud y complejidad silábica, identificar palabras a partir de una sílaba inicial y final dada, cambiar sílabas a las palabras para formar otras nuevas, reconocer objetos del entorno próximo según determinados fonemas, sustituir, omitir y clasificar palabras en función de la posición inicial, medial y final de los fonemas. Para ello, se utilizaron las rutinas "veo - pienso - me pregunto" y "antes pensaba - ahora pienso" favoreciendo la capacidad de análisis y reflexión cognitiva.

Para el aprendizaje del conocimiento alfabético se presentaron los títulos de los textos narrativos trabajados en clase, los nombres de sus personajes, así como la representación de las grafías a partir de vocabulario del entorno próximo y de los nombres de los propios compañeros de clase mediante el apoyo de organizadores visuales de las rutinas indicadas. Por ejemplo, mediante la rutina color-símbolo-imagen cada estudiante elegía un color que era el que asociaba a su nombre, un símbolo que lo representase y la imagen gráfica de la primera letra. En todas las dinámicas se potenciaba la combinación del trabajo individual y cooperativo, estas últimas moderadas por los responsables de cada equipo, siempre con la supervisión del docente con la finalidad de que se potenciase la capacidad de análisis, la reflexión conjunta, así como la puesta en común de las aportaciones e hipótesis que sobre el sistema de la escritura se iban generando, todo ello con el propósito de fomentar la expresividad verbal y el desarrollo del lenguaje escrito de los estudiantes.

\section{Resultados}

Con la finalidad de analizar el cambio en las variables del estudio se realizaron análisis descriptivos (medias y desviaciones típicas) con los datos obtenidos en los test administrados en la fase pretest, postest y en la diferencia postest-pretest, además de análisis de varianza con las puntuaciones pretest (MANOVA, ANOVA) y análisis de covarianza (MANCOVA, ANCOVA) de las diferencias encontradas postest-pretest en experimentales y control en las variables medidas antes y después de la intervención. Dichos análisis se realizaron con el programa SPSS 19.0. Además, se calculó el tamaño del efecto (d de Cohen) (pequeño < .50; moderado .50-.79; grande 2.80 ). Los resultados del MANOVA pretest para el conjunto de variables pusieron de relieve que antes de la intervención no existían diferencias significativas entre experimentales y control, $F(1,147)=1.34, p>$.05. Sin embargo, los resultados del MANCOVA de las diferencias postest-pretest, utilizando las puntuaciones pretest como covariables, fueron significativos: $F(1,147)=3.21, p<.05$. Estos datos indican que el programa de intervención tuvo un efecto significativo. Para analizar el cambio en cada variable se realizaron análisis descriptivos y de varianza, los cuales se presentan en la tabla 1. 


\section{Cambios en el desarrollo del lenguaje oral}

Con el propósito de evaluar el impacto del programa en el lenguaje oral, se analizaron los cambios en las puntuaciones obtenidas en el test PLON-R. EI MANOVA pretest realizado con el conjunto de las tres variables medidas (forma, contenido y uso) no evidenció diferencias significativas en la fase pretest entre experimentales y control, $F(1,147)=2.76, p$ > .05. Sin embargo, se encontraron diferencias significativas en el MANOVA postest-pretest, $F(1,147)=3.51, p<.01$, al igual que en el MANCOVA postest-pretest, $F(1,147)=3.26, p<.01$. Como se puede observar en la tabla 1 , en la variable forma, la muestra experimental obtiene un incremento $(M=.94)$, superior al conseguido por el grupo control $(M=.21)$. Los resultados del ANOVA pretest pusieron de manifiesto que en esta fase no había diferencias significativas entre experimentales y control, $F(1,147)=.452, p>$.05. Sin embargo, los datos del ANCOVA de las diferencias postest-pretest constataron resultados significativos, $F(1,147)=6.34, p<.01$. El tamaño del efecto fue moderado. En la variable uso se constatan de igual modo incrementos superiores en los experimentales $(M=1.05)$ frente a los del grupo control $(M=.19)$. Los resultados del ANOVA pretest reflejaron que a priori no existían diferencias significativas entre ambas condiciones, $F(1,147)=.523, p<.05$, realizándose un ANCOVA de las diferencias postest-pretest que indicó diferencias significativas, $F(1,147)=8.47, p<.001$. El tamaño del efecto fue moderado. Estos datos ponen de relieve una importante mejora del desarrollo del lenguaje oral atribuible al programa de intervención, que se observa en el incremento significativo de los componentes de la forma y uso.

\section{Cambios en la conciencia fonológica}

Con el objeto de analizar la eficacia del programa en el desarrollo de la conciencia fonológica, se estudiaron los cambios en las puntuaciones obtenidas en el test PECO. EI MANOVA pretest no evidenció diferencias significativas entre experimentales y control, $F(1,147)=2.36, p>.05$; sin embargo, los resultados del MANCOVA postest-pretest, $F(1,147)=1.43, p<.05$, confirmaron diferencias significativas entre ambas condiciones. No obstante, el análisis de cada variable de forma independiente únicamente ratificó diferencias en la conciencia silábica; la muestra experimental obtiene un incremento $(M=.71)$ superior al conseguido por el grupo control $(M=$.37). Los resultados del ANOVA pretest pusieron de manifiesto que en esta fase no había diferencias significativas entre experimentales y control, $F(1,147)=.263, p>$.05. Sin embargo, los datos del ANCOVA de las diferencias postest-pretest constataron resultados significativos, $F(1,147)=6.24, p<.01$. El tamaño del efecto fue pequeño. En la variable conciencia fonémica, se observó un aumento mayor en los experimentales $(M=1.147)$ que en los del grupo control $(M=64)$, y el ANCOVA postest-pretest puso de relieve diferencias estadísticamente significativas entre condiciones, $F(1,147)=11.27, p<.001$. El tamaño del efecto fue moderado. Esto pone de relieve una mejora de la capacidad para tomar conciencia de las unidades silábicas y fonémicas de las palabras atribuible al programa de intervención. 


\section{Cambios en los procesos cognitivos de la escritura}

Para evaluar si el programa fue eficaz en el desarrollo de los procesos cognitivos que intervienen en el aprendizaje de la escritura se analizaron los cambios en las puntuaciones logradas en el test PROESCRI Primaria. EI MANOVA pretest realizado para el conjunto de las variables de la prueba puso de manifiesto que no existían diferencias significativas en la fase pretest entre experimentales y control, $F(1,147)=2.23, p>$.05. Sin embargo, se encontraron diferencias significativas en el MANOVA postest-pretest, $F(1,147)=2.62, p<.01$, al igual que en el MANCOVA postest-pretest, $F(1,147)=3.61, p<.01$. Como se puede observar en la tabla 1 , en la variable generar palabras el grupo experimental obtiene una mejora $(M=.89)$ mayor que la conseguida por el grupo control $(M=.19)$. Los resultados del ANOVA pretest señalaron que en esta fase no había diferencias significativas entre experimentales y control, $F(1,147)=.134, p>$.05. Sin embargo, los datos del ANCOVA de las diferencias postest-pretest indicaron resultados significativos, $F(1,147)=8.27, p<.01$. El tamaño del efecto fue moderado. En la variable escritura de palabras con distinta longitud y familiaridad se constatan de igual modo incrementos superiores en los experimentales $(M=.98)$ frente a los del grupo control $(M=.16)$. Los resultados del ANOVA pretest señalaron que a priori no existían diferencias significativas entre ambas condiciones, $F(1,147)=.416, p<.05$, realizándose un ANCOVA de las diferencias postest-pretest que indicó diferencias significativas, $F(1,147)=15.28, p<.001$. El tamaño del efecto fue moderado. También se produjo una mejora tendencialmente significativa en escritura de palabras con ortografía arbitraria, $F(1,147)=2.86, p<.05$, con aumentos superiores en los experimentales $(M=.95)$ que en los de control $(M=.14)$, así como en la escritura de pseudopalabras, $F(1,147)=3.47, p<.05$, con un aumento mayor en los experimentales $(M=.97)$ que en los pertenecientes al grupo control $(M=.15)$. Estos datos ponen de manifiesto una mejora del aprendizaje de la escritura atribuible al programa de intervención puesto en práctica.

\section{Discusión y conclusiones}

El objetivo de este estudio era comprobar el efecto que la intervención en el desarrollo de las habilidades que favorecen el aprendizaje de la escritura mediante dinámicas que potencian la capacidad de pensar a través de las rutinas de pensamiento presenta en el proceso de adquisición del código escrito en las primeras edades. Los resultados obtenidos señalan que la instrucción en estas habilidades, a través de la implementación en el aula de las rutinas del pensamiento, favorece la reflexión y el aprendizaje sobre el proceso de construcción del sistema de la escritura.

Analizando los datos del presente estudio se comprueba que el grupo experimental ha mejorado de manera significativa respecto al grupo control en la capacidad expresiva a nivel verbal, lo que se ha puesto de manifiesto tanto en el componente de la forma, en concreto respecto al desarrollo fonológico y morfosintáctico, como en la funcionalidad en el uso de la lengua, al producirse mejoras en la capacidad comunicativa en situaciones de carácter 


\section{TABLA 1}

Medias y desviaciones típicas en lenguaje oral, conciencia fonológica y escritura de letras, palabras y pseudopalabras, y resultados del análisis de varianza y covarianza para el grupo experimental y control

\begin{tabular}{|c|c|c|c|c|c|c|c|c|c|c|c|c|c|}
\hline & \multicolumn{5}{|c|}{$\begin{array}{l}\text { GRUPO EXPERIMENTAL } \\
(\mathrm{n}=180)\end{array}$} & \multicolumn{5}{|c|}{$\begin{array}{l}\text { GRUPO CONTROL } \\
\quad(n=176)\end{array}$} & \multicolumn{3}{|c|}{$\begin{array}{l}\text { EXPERIMENTAL - CONTROL } \\
(n=356)\end{array}$} \\
\hline & Pre & & Post & & Post-Pre & Pre & & Post & & Post-Pre & $\begin{array}{c}\text { Anova } \\
\text { Pretest }\end{array}$ & $\begin{array}{l}\text { Anova } \\
\text { Postest- } \\
\text { Pretest }\end{array}$ & $\begin{array}{c}\text { Ancova } \\
\text { Postest-Pretes }\end{array}$ \\
\hline ARIABLES & $M$ & $D T$ & $M$ & $D T$ & $M \quad D T$ & M & $D T$ & $M$ & $D T$ & $M \quad D T$ & $d \underset{(1,147)}{F}$ & $\underset{(1,147)}{F}$ & $\underset{(1,147)}{F}$ \\
\hline
\end{tabular}

PLON-R

\begin{tabular}{llllllllllllllllll} 
Forma & 1.49 & .27 & 2.43 & .32 & .94 & .71 & 1.51 & .31 & 1.72 & .62 & .21 & .23 & .32 & .452 & $4.25^{* *}$ & $6.34^{* *}$ & .37 \\
Contenido & 1.46 & .42 & 2.49 & .51 & 1.03 & .42 & 1.48 & .48 & 2.43 & .38 & .95 & .17 & .12 & .024 & 1.30 & 4.15 & .15 \\
Uso & 1.52 & .23 & 2.57 & .63 & 1.05 & .62 & 1.53 & .25 & 1.72 & .41 & .19 & .62 & .78 & .523 & $2.45^{* * *}$ & $8.47^{* * *}$ & .83 \\
\hline
\end{tabular}

PECO

$\begin{array}{llllllllllllllllll}\text { C. Silábica } & 2.83 & .41 & 3.54 & .42 & .71 & .53 & 2.79 & .56 & 3.16 & .42 & .37 & .52 & .31 & .263 & 4.38^{*} & 6.24^{*} & .34\end{array}$

$\begin{array}{lllllllllllllllllll}\text { C. Fonémica } & 2.36 & .72 & 3.85 & .51 & 1.49 & .82 & 2.37 & .24 & 3.01 & .74 & .64 & .63 & .67 & .317 & 9.36^{* * *} & 11.27^{\star * *} & .71\end{array}$

PROESCRI

$\begin{array}{llllllllllllllllll}\text { Escritura letras } & 1.86 & .51 & 2.52 & .51 & .66 & .42 & 1.88 & .41 & 2.50 & .32 & .62 & .56 & .12 & .296 & .163 & .008 & .14\end{array}$ $\begin{array}{llllllllllllllllll}\text { Generar palabras } & 1.54 & .63 & 2.43 & .32 & .89 & .81 & 1.52 & .37 & 1.71 & .46 & .19 & .48 & .54 & .134 & 4.78^{* *} & 8.27^{* *} & .62\end{array}$

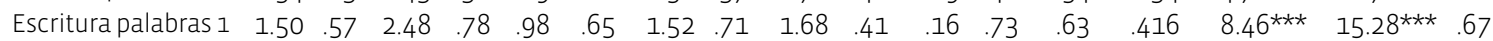

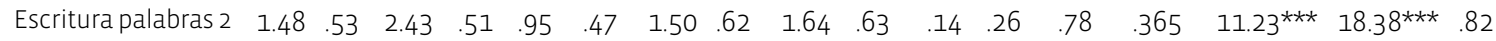
$\begin{array}{llllllllllllllllll}\text { Escritura } & 1.42 & .68 & 2.39 & .82 & .97 & .62 & 1.43 & .48 & 1.58 & .82 & .15 & .39 & .82 & 5.42 & 12.42^{\star * *} & 1472^{\star * *} & .84\end{array}$ pseudopalabras

${ }^{*} p<.05 \quad * * p<.01 \quad * * * p<.001$

pragmático en el aula. Estos aportes coinciden con las afirmaciones de otros autores que defienden la relevancia del desarrollo del lenguaje oral en el proceso de adquisición del lenguaje escrito (Gutiérrez-Fresneda, 2017; Gutiérrez-Fresneda y Díez, 2017; Núñez y Santamaría, 2014), lo que indica que la puesta en práctica desde las primeras edades de propuestas que favorezcan el desarrollo de la capacidad comunicativa oral facilitará el acceso al aprendizaje de la escritura de los estudiantes que se inician en el proceso de alfabetización.

De igual modo, el alumnado del grupo experimental ha mejorado de manera significativa respecto al grupo control en la capacidad para manejar y tomar conciencia de las unidades del lenguaje hablado, lo que refleja que el entrenamiento en las habilidades que contribuyen a la mejora de la conciencia fonológica constituye un aspecto de gran relevancia en la fase inicial del aprendizaje de la escritura favoreciendo su adquisición, lo que constataría los beneficios que el desarrollo de los procesos fonológicos presentan en la adquisición del sistema de la escritura (Gutiérrez, 2017; Gutiérrez y Díez, 2018). De igual modo, es relevante destacar que, de los distintos niveles que integran la conciencia fonológica, la capacidad para manejar las unidades mínimas de las palabras es el aspecto que más vinculación presenta con el apren- 
dizaje de la escritura, en comparación con la toma de conciencia de las unidades silábicas, hallazgo que es consistente con los resultados obtenidos en estudios precedentes (Defior y Serrano, 2011; Gutiérrez, 2017).

Respecto a los procesos de aprendizaje de la escritura, los resultados recogidos señalan que aunque no se producen diferencias significativas en el aprendizaje del conocimiento de las letras de nuestro código, el programa de intervención sí contribuyó en la mejora de las destrezas que intervienen en la capacidad para escribir palabras de distinta longitud y familiaridad, con un tamaño del efecto moderado, así como en la escritura de palabras sin errores ortográficos y en la correcta escritura de pseudopalabras, con un tamaño del efecto grande. Todo esto refleja que los alumnos participantes en el programa logran una mejoría relevante tanto en el procesamiento fonológico como ortográfico que les permite acceder a la representación de las palabras de forma rápida y precisa debido a una mayor automatización de las reglas de correspondencia fonema-grafema, las que a su vez posibilitan el desarrollo de las habilidades ortográficas que están asociadas con la adecuada escritura de las palabras, datos que son consistentes con los hallazgos encontrados en otros trabajos anteriores (González, Cuetos, Vilar y Uceira, 2015; Gutiérrez-Fresneda, 2017; Suárez-Coalla, García de Castro y Cuetos, 2013).

Este trabajo pone de manifiesto que las situaciones que se generan a partir de los organizadores gráficos de las rutinas de pensamiento contribuyen entre otros aspectos a desarrollar nuevas ideas, reflexionar de manera conjunta sobre las hipótesis que se generan sobre el código escrito, secuenciar la información en función de los objetivos de escritura que se establecen, expresar la información de manera ordenada, así como ofrecer soluciones creativas a diferentes propuestas sobre el lenguaje escrito. Una explicación de estos progresos puede deberse a la ayuda que proporcionan los organizadores gráficos y las preguntas guía de las rutinas del pensamiento ya que contribuyen a la toma de conciencia sobre el proceso de construcción de la escritura, así como al progreso que los compañeros efectúan de manera conjunta. Estos aportes coinciden con las aseveraciones de otros autores que destacan los beneficios de las habilidades de pensamiento en el aprendizaje de los contenidos escolares y sobre los procesos de composición escrita en estudiantes de niveles superiores (Gallardo y Novillo, 2017; Gutiérrez-Fresneda y Díez-Mediavilla, 2017; Gutiérrez-Fresneda, 2018; Toro y Arguis, 2015).

Los datos hallados en esta investigación permiten igualmente deducir que un factor que puede influir de manera positiva en el proceso de adquisición de este aprendizaje es el trabajo interactivo que se produce entre los propios compañeros a través de las situaciones dialógicas que se generan mediante las rutinas del pensamiento, ya que este tipo de dinámicas contribuyen a la puesta en común sobre las características del sistema de la escritura. Mediante este recurso pedagógico se posibilita que los distintos compañeros hagan explícitas las estrategias que van utilizando para acceder al sistema de la escritura a través de las aportaciones que se realizan a través de la reflexión individual y colectiva, lo que puede considerarse una 
medida eficaz para la adquisición de estrategias de aprendizaje de la escritura. De hecho, son varios los estudios que han evidenciado los beneficios del trabajo compartido en el proceso de aprendizaje (Coulson y Harvey, 2013), por lo que un factor que también puede contribuir al proceso de alfabetización infantil puede ser el fomento de las situaciones de interacción conjunta en cuanto que permiten intensificar el desarrollo de los distintos componentes del lenguaje hablado. Este hecho resulta de interés puesto que mediante la generación de dinámicas comunicativas en la práctica del aula se puede contribuir a compensar las carencias que determinados alumnos presentan en la capacidad expresiva en las edades tempranas, lo cual presenta una gran relevancia en el proceso de adquisición del sistema de la escritura (Guarneros y Vega, 2014; Gutiérrez-Fresneda y Díez-Mediavilla, 2017).

En resumen, este estudio es relevante ya que contribuye a la facilitación de los procesos de aprendizaje del código escrito, por lo que a nivel práctico se sugiere el diseño de propuestas didácticas basadas en el desarrollo de las habilidades prescritoras a través de las rutinas del pensamiento junto con el apoyo de organizadores gráficos en las que se potencie el desarrollo léxico, la generación de hipótesis sobre el proceso de construcción de la escritura, el análisis reflexivo y el pensamiento en voz alta sobre este aprendizaje, ya que de este modo se facilita el acceso al sistema de la escritura. Una limitación de este trabajo es que no se atendió a determinadas variables, como el grado de motivación hacia el aprendizaje del lenguaje escrito de los estudiantes, las prácticas escritoras realizadas en el ámbito familiar, así como los niveles de escolarización de los padres, que de igual modo pueden resultar determinantes en el proceso de aprendizaje de la escritura. Este estudio abre también nuevas líneas de investigación, como es el caso de analizar si mediante la utilización de las rutinas de pensamiento se puede fomentar el desarrollo cognitivo de los procesos superiores que están implicados e intervienen directamente en el aprendizaje de la composición escrita.

\section{Bibliografía citada}

Aguinaga, Gloria, María luisa Armentia, Ana Fralle, Pedro Olangua y Nicolás Útiz, 2004: Prueba de Lenguaje Oral de Navarra-Revisada (PLON-R), Fondo de Publicaciones del Gobierno de Navarra.

Araya, Natalia, 2014: "Las habilidades del pensamiento y el aprendizaje significativo en matemática de escolares de quinto grado en Costa Rica", Revista Electrónica Actualidades Investigativas en Educación 14 (2), 1409-4703.

Aznar, Inmaculada, e Ignacio LaITon, 2017: "Desarrollo de Habilidades Básicas de Pensamiento Crítico en el Contexto de la Enseñanza de la Física Universitaria”, Formación Universitaria $10(1), 71-78$.

Artiles, Ceferino, y Juan Jiménez, 2007: PROESCRI Primaria. Prueba de evaluación de los procesos cognitivos en la escritura, Consejería de Educación, Cultura y Deportes del Gobierno de Canarias. 
BÁez, Javier, y Javier OnRUBiA, 2016: "Una revisión de tres modelos para enseñar las habilidades de pensamiento en el marco escolar”, Perspectiva Educacional. Formación de Profesores 55 (1), 94-113.

Beas, Josefina, Josefina Santa Cruz, Paulina Thomsen y Soledad Utreras, 2001: Enseñar a pensar para aprender mejor, Ediciones Pontificia Universidad Católica de Chile.

Boodt, Gloria, 1984: "Critical listeners become critical readers in remedial reading class", The Reading Teacher 37 (4), 390-394.

BRAvo, Luis, 2016: "The learning of written language and the sciences of reading. A limit between cognitive psychology, neuroscience and education", Limit. Interdisciplinary Journal of Philosophy and Psychology 11 (36), 50-59.

Bravo, Luis, Malva Villalón y Eugenia Orellana, 2006: "Predictibilidad del rendimiento en la lectura: una investigación de seguimiento de primer a tercer año", Revista Latinoamericana de Psicología 38 (1), 9-20.

Coulson, Debra, y Marina HaRvey, 2013: "Scaffolding student reflection for experience-based learning: A framework”, Teaching in Higher Education 18 (4), 401-413.

Defior, Silvia, y Francisca Serrano, 2011: "La conciencia fonémica, aliada de la adquisición del lenguaje escrito”, Revista de Logopedia, Foniatría y Audiología 31 (1), 2-13.

Dickinson, David, 2011: "Teachers' language practices and academic outcomes of preschool children", Science 333 (6045), 964-967 [http://doi.org/10.1126/ science.1204526].

Díez, Antonio, Luis Güemes y María Molina, 2018: "Preconceptions and education of the reading and writing in the initial training of the students of Magisterio", Investigaciones Sobre Lectura 9, 105-120.

Espejo, Patricia, Raúl Gutiérrez, Dolores Llambés y Beatriz Vallejo, 2008: Avanza. Programa para el desarrollo de las habilidades escolares básicas. Iniciación al aprendizaje de la lectoescritura. Habilidades fonológicas I, ECU.

Espejo, Patricia, Raúl Gutiérrez, Dolores Llambés y Beatriz Vallejo, 2015: Avanzados. Programa para el desarrollo de las habilidades escolares básicas. Iniciación al aprendizaje de la lectoescritura. Habilidades fonológicas II, ECU.

FerReIRo, Emilia, 1979: Los sistemas de escritura en el desarrollo del niño, Siglo XXI Editores.

Ferreiro, Emilia, y Ana Teberosky, 1988: Los sistemas de escritura en el desarrollo del niño, Siglo XXI Editores. 
Gallardo, Jesús Manuel, y Miguel Ángel Novillo, 2017: "Aprender Historia con rutinas de pensamiento. CLIO", History and History Teaching 43, 153-176.

GonzAlez, Rosa María, Fernando Cuetos, Juan Vilar y Eva Uceira, 2015: “Efectos de la intervención en conciencia fonológica y velocidad de denominación sobre el aprendizaje de la escritura”, Aula Abierta 43, 1-8.

González, Rosa María, Silvia Lópe, Juan Vilar y Alfredo Rodriguez, 2013: "Study of reading predictors", Journal of research in education 11 (2), 98-110.

Graham, Steve, 2006: “Writing”, en P. Alexander y P. Winne (eds.): Handbook of educational psychology, Mahwah: Erlbaum, 457-478.

Guarneros, Esperanza, y Lizbeth VegA, 2014: "Habilidades lingüísticas orales y escritas para la lectura y escritura en niños preescolares”, Avances en Psicología Latinoamericana 32 (1), 21-35.

Gutiérrez, Raúl, 2017: "Facilitators of the learning process of writing in early ages", Anales de Psicología 33 (1), 32-39.

GutiérREZ, Raúl, 2018: "Habilidades favorecedoras del aprendizaje de la lectura en alumnos de 5 y 6 años", Revista Signos 51 (96), 45-60.

GutiéRREZ-FresnedA, Raúl, 2017: “Efectos de la comunicación dialógica y la conciencia fonológica en el aprendizaje inicial de la escritura de palabras en español”, Onomázein 37, 170-187 [doi: 10.7764/onomazein.37.10].

GutiérRez-Fresneda, Raúl, 2018: “Las destrezas del pensamiento y el aprendizaje compartido para la mejora de la composición escrita”, Estudios sobre Educación 34, 263-281.

Gutiérrez-FresnedA, Raúl, y Antonio Diez, 2017: “Componentes del lenguaje oral y desarrollo evolutivo de la escritura en las primeras edades", Álabe: Revista de Investigación sobre Lectura y Escritura 16, 1-15 [doi: 10.15645/Alabe2017.16.3].

Gutiérrez-Fresneda, Raúl, y Antonio Díez, 2018: "Conciencia fonológica y desarrollo evolutivo de la escritura en las primeras edades”, Educación XX1 21 (1), 395-416.

Gutiérrez-Fresneda, Raúl, y Antonio Diez-Mediavilla, 2017: “Efectos de la comunicación dialógica en la mejora de la composición escrita en estudiantes de Primaria”, Teoría de la Educación. Revista Interuniversitaria 29 (2), 41-59.

Gutiérrez-Fresneda, Raúl, Antonio Diez y Elena Jiménez-Pérez, 2017: "Estudio longitudinal sobre el aprendizaje lector en las primeras edades", Revista de Educación 378, 28-49. 
Llamazares, María Teresa, María Dolores Alonso-Cortés y Susana Sánchez, 2015: "Factores que influyen en el aprendizaje de la comprensión lectora y de la composición escrita: tres estudios realizados en aulas de Educación Infantil y Educación Primaria”, Revista Investigaciones sobre Lectura 3, 67-82.

Montesdeoca, Deborah, María Elena Gómez-ParRa y Roberto Espejo, 2020: "Estrategias de enseñanza de la comprensión lectora aplicadas y percibidas: un estudio con docentes y estudiantes de cuarto grado de educación básica de Manabí-Ecuador", Investigaciones sobre Lectura 14, 165-180.

Nemirovsky, Miriam, 1999: Sobre la enseñanza del lenguaje escrito... y temas aledaños, Paidós.

NorRIS, Stephen, 1985: "Synthesis of research on critical thinking", Educational Leadership $42(8), 40-46$.

Núñez, María Pilar, y María Santamarí, 2014: "Prerrequisitos para el proceso de aprendizaje de la lectura y la escritura: conciencia fonológica y destrezas orales de la lengua”, Lengua y Habla 18, 72-92.

Peña, Olga, y Aura Quintero, 2016: "La escritura como práctica situada en el primer ciclo: promoción de procesos cognitivos y metacognitivos", Cuadernos de Lingüística Hispánica 28, 189-206.

Ramos, José Luis, e Isabel Cuadrado, 2006: Prueba para la Evaluación del Conocimiento FonológicO. PECO, EOS.

RAPP, Martha, 1988: "Developing critical thinking with the directed reading-thinking activity", The Reading Teacher 41 (6), 526-533.

Sáz, Carlos, y Silvia Fernandez, 2012: "Pensamiento crítico y aprendizaje basado en problemas Cotidianos”, Revista de Docencia Universitaria 10 (3), 325-346.

Schatschneider, Christopher, Jack Fletcher, David Francis, Coleen Carlson y Barbara Foorman, 2004: "Kindergarten prediction of reading skills: A longitudinal comparative analysis", Journal of Educational Psychology 96 (2), 265-282.

SHARE, David, 2004: "Knowing letter names and learning letter sounds: A causal connection", Journal of Experimental Child Psychology 86, 1-31.

Suarez-Coalla, Paz, Marta García de Castro y Fernando Cuetos, 2013: "Variables predictoras de la lectura y la escritura en castellano", Infancia y Aprendizaje 36 (1), 77-89.

Swartz, Robert, y David Perkins, 1989: Teaching thinking issues and approaches, Midwest Publishers. 
Teberosky, Ana, y Claudia Portilla, 2011: “Los 'contrarios' en el aprendizaje inicial del lenguaje escrito”, Cultura y Educación 23 (4), 515-531.

Toro, Alberto, y Marta Arguis, 2015: “Metodologías Activas”, A Tres Bandas 38, 69-77.

ValenzUela, Jorge, 2007: "Habilidades de pensamiento y aprendizaje profundo", Revista Iberoamericana de Educación 46 (7), 1-9. 